Editorial

\title{
Endogenous toxins as disease initiating events: future targets of drug discovery research
}

Volume 2 Issue 6 - 2016

\section{Introduction}

I(SV) joined pharmaceutical industry as a senior scientist at a time when the productivity was shrinking and mergers and acquisitions were becoming the mainstream focus for the industry to sustain. At that time, there were employees who had worked in the same company for years and retired. Today, due to many patent expiries, escalating costs of drug discovery and development ( 4 billion USD for one drug to reach market), increasing regulatory barriers, and shrinking productivity - pharmaceutical industry is experiencing a roller-coaster ride and unable to sustain their R\&D programs for new drug discovery. ${ }^{1-5}$ Therefore, for the current generation of employees job security is the glory of the past and they are changing jobs often to find professional growth. So, what is happening? Why is the productivity shrinking? Why is industry not able to sustain and come up with new drugs?

The answer to the above questions primarily lies in the complexity of biology. Targeted drug discovery has become the mainstream effort of R\&D in the last two decades. However, due to redundancy of network pathways and inherent adverse pharmacology of many targets, it is proving difficult for discovering and developing new drug candidates using a single target. About $87 \%$ of phase III clinical trials fail due to either lack of efficacy $(66 \%)$ or safety $(21 \%)$. Between 2007 and 2010, out of 83 molecules that failed in phase III trials, oncology (28\%) and CNS disorders (18\%) topped the list of failures (2.3).

Due to the poor productivity, pharmaceutical industry witnessed several paradigm shifts in the last two decades. Cutting edge technologies in molecular biology, analytical instrumentation and, robotics have helped screen several millions of compounds. However, these efforts only resulted in escalating costs of drug discovery and development with no improvement in productivity. In order to reduce the development costs, many pharmaceutical companies off-shored their drug development operations to countries like China and India, to name a few. Academic collaborations with MIT, Harvard and many other premium institutes is still in early stages and has not come to fruition. ${ }^{6,7}$ In our opinion, failure in pharmaceutical industry is primarily due to flawed target selection as most are at the late stages of the disease. These targets are simply passenger or casual targets in the cascade of many other events happening in a proliferating cell. We are also of the opinion that target selection at early initiation phase of a disease is more critical in causation of the disease. So, what are these early stage targets? What is the likely hood of discovering these early targets? Will targeting these early targets in drug discovery help in preventing the progression of the disease? We would like to try to answers to these questions below

\section{Are Endogenous Toxins Responsible for Development of Incurable Chronic Diseases?}

Perhaps, the major issue for the flawed selection of drug targets in

\author{
Subrahmanyam Vangala, Prema Kolachana \\ InCan Solutions Pvt Ltd, Nova Scotia, Canada
}

Correspondence: Subrahmanyam Vangala, InCan Solutions Pvt Ltd, Halifax, Nova Scotia, Canada, Tel I-908-905-0556,

Email subrahmv_2000@yahoo.com

Received: July 06, 2016 | Published: July II, 2016

drug discovery and development is over-simplification of biological networks and underestimation of the complexity of biology. Chronic diseases require multiple, sequential hits in the biochemical pathways of cellular homeostasis which in turn leads to fatal disease. For example, in inborn errors of metabolism, most children are born with a deficiency of a single enzyme or protein, but as they grow, they develop serious fatal complications eventually resulting in death ${ }^{8,9}$ The continued accumulation of one metabolite due to the deficiency of an enzyme or protein, becomes the primary endogenous toxin. This in turn ensues a cascade of secondary endogenous toxins and so on, thus resulting in disease progression to an irreversible stage. ${ }^{8,9} \mathrm{~A}$ simple example is insulin deficiency or deficiency in uptake receptors of glucose in liver and muscle can increase the accumulation of glucose in the systemic circulation. This accumulation of glucose, if not reversed, can result in severe diabetic complications including, gangrene, nephropathy, retinopathy and stroke, to name a few. ${ }^{10,11}$ Host of genetic and epigenetic events have been related to diabetic complications that follow hyperglycemia. ${ }^{11,12}$ Nevertheless, the simple reason for these complications is the chronic accumulation of unutilized glucose which is highly reactive and acylates proteins in the vicinity resulting in irreversible cellular damage. ${ }^{10}$ Chronically, this damage can create cascade of events resulting in the induction of inflammatory processes causing further damage and eventually causing varied diabetic complications (Figure 1). Recent studies indicate IL1 beta, a pro inflammatory cytokine increases the uptake of glucose into smooth aortic muscle cells which stimulate the pentose phosphate pathway. ${ }^{13}$ High levels of NADPH generated are metabolized via NADPH oxidase setting a stimulus for oxidative stress. Genome Wide Association Studies (GWAS) revealed that type 2 diabetes is a polygenic disease and there are hundreds of different gene variants working in a complex interplay influencing pancreatic beta cell function. ${ }^{11,12}$ Nevertheless, today, diabetes is well controlled due to availability of various recombinant human insulin forms, oral medications, and regular exercise, prevent glucose accumulation in the systemic circulation and diabetic complications. Glucose is thus 
the primary, initiating Endogenous Toxin. Several such Endogenous Toxins have been known for various neonatal inborn diseases where the deficiency of an enzyme/protein is known. ${ }^{8,9}$

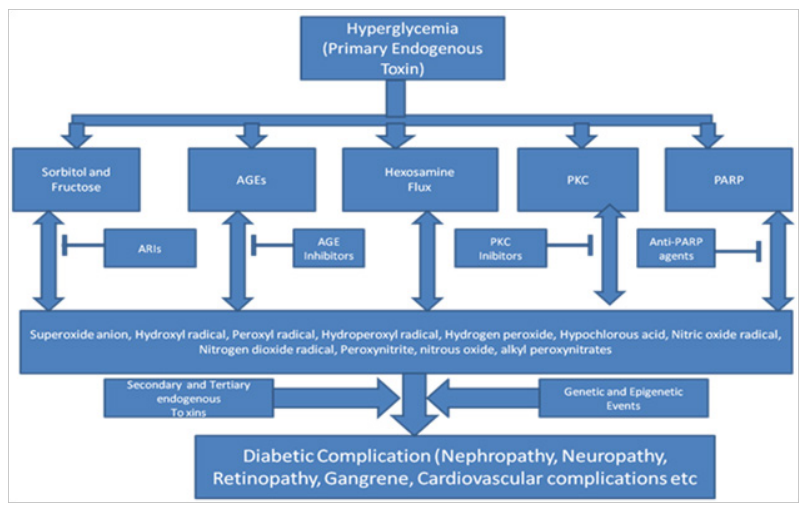

Figure I Consequences of hyperglycemia (AGEs: Advanced Glycations Endproducts, PKC; Protein Kinase C, PARP: Poly ADP Ribose Polymerase).

\section{What are Endogenous Toxins of cancer?}

Cancer is thought to arise due to mutagenic events either through direct action on DNA by mutagens [eg, benzo[a]pyrene diolepoxide] or indirect action via endogenous mutants [eg, hydroxyl radical, lipid peroxides] or via epigenetic mechanisms [eg., spindle damage during cell division]. ${ }^{15-20}$ Cancer has at least four different steps, namely initiation, promotion, progression and metastasis (Figure 2) - spanning over two to three decades. The first step is initiation (DNA damage with a mutagenic event in a normal healthy cell), second step is promotion (mutated cell is stimulated to proliferate and differentiate from normal cell), the third step is progression (wildly mutated cells further proliferate to form tumor with several mutations) and the fourth stage metastasis (spreading to other organs).

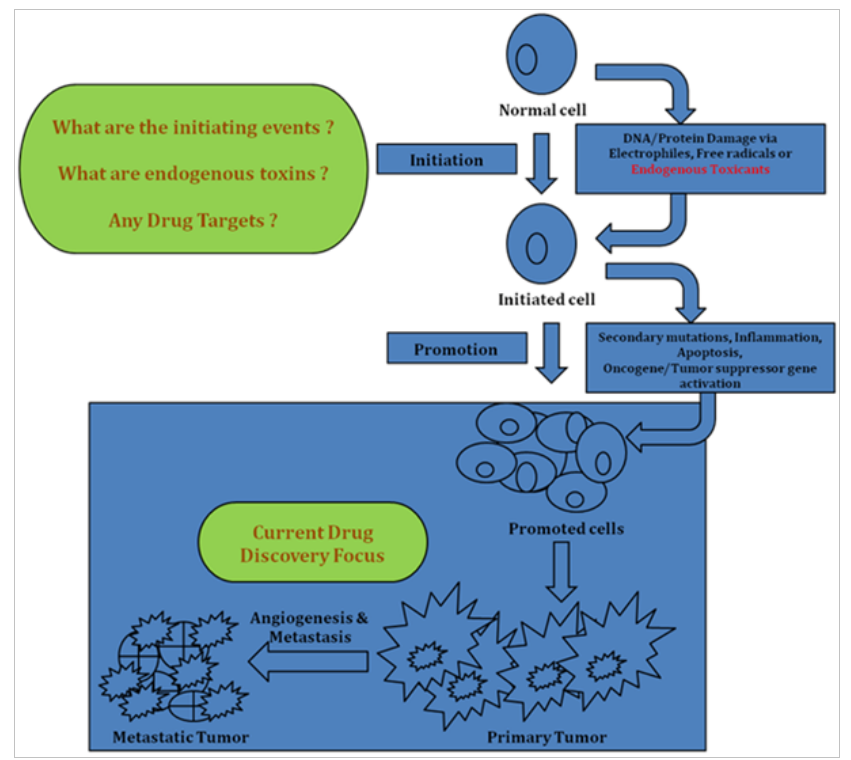

Figure 2 Initiation, promotion, progression and metastasis phases of cancer.

Last 10-15years, extensive research was conducted to understand cancer genome landscape in the hope of discovering novel, new drugs for appropriate therapeutic interventions. ${ }^{21}$ Many solid tumors contain 30 to 70 mutations that alter the amino acid sequences of the proteins encoded by the affected genes..$^{21,22}$ In addition, greater than $99.9 \%$ of the alterations in tumors including point mutations, copynumber alterations, translocations, and epigenetic changes distributed throughout the genome, not just limited to the coding regions which are unlikely cancer initiating events. They are simply passenger or casual changes that indicate the time elapsed between successive clonal expansions. ${ }^{21}$ When do these mutations occur? Taking colorectal cancers (CRCs) as an example, the spectrum of somatic mutations contributing to the pathogenesis of CRC is very extensive, mutations harboring several genes including APC, BRCA1/2, BRAF, KRAS, MLH1, MSH2, MSH6, NRAS, PIK3CA, SMAD4, TP53 and several other genes have been described in the literature. ${ }^{21-27}$ Sixtynine genes were highlighted as relevant to the pathogenesis of CRCs, and individual CRCs harbored an average of nine mutant genes per tumor. In addition, each tumor studied had a distinct mutational gene signature..$^{22}$ Certainly, all these gene mutations are likely the end events but not early events in colorectal cancers. Nevertheless, it appears that gate-keeping initiating events in the colon most often occur in the APC gene that offers growth advantage for the initiated cell. Nevertheless, the endogenous toxin that initiates the mutation of APC gene is still not known. Thus, the heterogeneity in cancer genomics, certainly makes standardized therapies for each mutant form is extremely difficult. The question should be asked is, what are the Endogenous Toxins that cause an initial mutation (eg. APC gene), which triggers series of other mutations, genome instabilities and other epigenetic changes.

Recent attempts to find association between Advanced Glycation Products (AGEs) and colorectal cancer in more than 1000 patients did not find any significant correlations. ${ }^{28,29}$ Many such studies applying metabolomics tools are in progress. Nevertheless, many of these studies are focusing on late stage events, similar to genomics. Systematic metabolomic approach to identify the initiating events in colorectal cancers and other cancers; so far have not been conducted. Any signature, either of genome, proteome or metabolome obtained at end stage disease will be not be predictive of disease initiating events. Future studies should focus on discovering critical Endogenous Toxins responsible for these cancers. Same strategy should be applied for various other diseases including neurodegenerative diseases. An early detection of disease can certainly improve discovery of drugs that can prevent the disease progression.

\section{Future challenges in applying OMICS technologies for a productive drug discovery research}

Advances in Analytical tools in Transcriptomics, Genomics, Proteomics and Metabolomics are certainly helping to understand the biological networks in various organs in humans. Understanding these networks is still at its infancy. About $99.5 \%$ of the DNA sequence is similar in all humans. ${ }^{30}$ However, genetically, no two individuals are similar. ${ }^{30}$ In 2011, citing the published research by Conrad et al., ${ }^{22}$ Scientific Daily stated: We are all mutants: First direct whole-genome measure of human mutation predicts 60 new mutations in each of us. ${ }^{31}$ As mentioned above, type 2 diabetes, cancer and many other diseases are polygenic with more than 100 genetic variants involved. Many genes are highly polymorphic and alleles occur at different frequencies in different human populations. ${ }^{32,33}$ Thus, the genetic fingerprinting is the key for understanding the individual differences.

Many drugs only work in a fraction of patients due to gene polymorphisms in the target protein or enzyme. ${ }^{22,30}$ Even if the drug works in some patients, the dose may vary due to genetic polymorphisms in drug metabolizing enzymes. ${ }^{34}$ If a drug $\mathrm{X}$ works well at $10 \mathrm{mg}$ dose in some patients, other patients may not respond until the dose is increased several fold higher. However, at such higher doses, the drug may also have adverse side effects limiting its 
use. Perhaps another drug Y, may work very well for those patients who are not responsive to drug X. So, for personalized and precision medicine to be effective - OMICS signatures have to be understood well, so Right Drug-Right Dose for each patient can be determined with precision.

Currently, the cost of sequencing a human genome is markedly reduced to $\$ 1000 /$ genome..$^{35}$ However, the mutational spectrum of genome is complicated and many times a single gene may have multiple mutations and thus diagnostic tools are still in infancy. So, sequencing human genome alone will not help in understanding disease pathways. The combination of genomics and metabolomics (and perhaps proteomics) in diseased (at different stages of disease) and normal humans (base control comparator) may offer promise in unraveling the Endogenous Toxins which initiate the disease process. Once these Endogenous Toxins involved in disease initiation are identified predicting and preventing of diseases may become the reality of future. Prophylactic intervention, at disease initiation stage, with a drug or neutraceutical could prevent progression maintaining normal hemeostasis. The genomic and metabolic signatures as early as at neonatal stage and periodically every year can help to identify the disease initiating events at an early stage, thus, chronic diseases can be prevented before they become full blown diseases.

\section{Metabolomic signatures as clinical chemistry tools to discover Endogenous Toxins}

Human metabolome (v3.0) has listed more than 37,000 metabolites with biofluid and tissue concentration data is known for over 6000 metabolites. ${ }^{35}$ Approximately, 4000 metabolites have been linked to various diseases. Using these data about 440 metabolite pathway networks have been described so far. Despite the advances in analytical tools, only $\sim 3000$ metabolites are supported with structural confirmation data using NMR, LC-MS-MS or GC-MS analysis. The future emphasis in drug discovery should understand these network pathways using genomics, proteomics and metabolomics. Metabolite signatures as biomarkers of efficacy and as extended clinical chemistry as biomarkers of toxicity will be a very powerful tool in disease treatment. ${ }^{8,937}$ Thus, OMICS research will improve our understanding the Endogenous Toxins in early phase of disease initiation and for appropriate therapeutic intervention.

\section{Conclusion}

Disease biology is highly complex with many redundant networks. For many chronic diseases, several multiple, sequential hits are required for disease to progress. Unlike diabetes, diseases like cancer and neurodegenerative disorders are still elusive to biologists. Current strategy involves targeting the disease in the late stages, which is proving to be futile and cost prohibitive. With this approach phase III trials are failing and few effective curative medicines are available. Majority of the diagnosis is done using histopathology techniques of biopsies, often coupled with CT Scans, MRI and screening for specific gene mutations. Especially, the Endogenous Toxins that are responsible for initiation of the vast majority of chronic diseases are still not known. If the first critical step of disease initiation process is known then the interception at that stage can prevent the progression of the disease. Currently, the only choice available is to slow down the progression and prolong survival for a few more years. This usually requires extensive hospitalization, treatment with very expensive drugs, ambulatory care, which is very expensive. Some medications, especially related to cancer which prolongs the survival are costing at $\$ 200,000$ or more/year which many patients cannot afford. ${ }^{30}$ Translational pharmacology \& toxicology with a focus on unraveling early and sequential events in disease events followed by appropriate prophylatic interventions should be the focus of drug discovery research in the future. ${ }^{37}$ Technological innovation using organ-, animal- or human-on-a-chip models can link the translation of in vitro and animal research to humans. ${ }^{38}$

Philosophically speaking, separating rhetoric from prevention, in reality, is a complex task. Many non-scientists ask me why don't we have cure for many diseases? Pharmaceutical industry is more interested in filling their pockets rather than coming up with cures? Yes, these questions are very relevant. However, my answer to these critics is to encourage funding for basic research. Secondly, patenting has become the main stream focus and thus basic research has been sidelined for the last twenty to thirty years. For example, nutraceuticals derived from herbal alternatives have not raised any interest in pharmaceutical industry because of limited patentability on such medicines. Let me conclude with the quote of Herman Biggs, an American pioneer in public health, who was the Commissioner of Health of New York State, published in a Monthly Bulletin in 1911.

'Disease is largely a removable evil. It continues to afflict humanity, not only because of incomplete knowledge of its causes and lack of adequate individual and public hygiene, but also because it is extensively fostered by harsh economic and industrial conditions and by wretched housing in congested communities. These conditions and consequently the diseases which spring from them can be removed by better social organization. No duty of society, acting through its governmental agencies, is paramount to this obligation to attack the removable causes of disease ... The provision of more and better facilities for the protection of the public health must come in the last analysis through the education of public opinion so that the community shall vividly realize both its needs and its powers ... The reduction of the death rate is the principal statistical expression and index of human and social progress ... Public health is purchasable' (Winslow 1929).

It is ironic after more than a century of this quote was stated; we are still in the infancy of understanding the complexity of human biology. Most diseases are due to environmental, dietary and different personal life style factors. If these underlying causes can be taken care of, then, majority of somatic diseases can be prevented and we can focus on those less fortunate with some genetically inherited diseases.

\section{Acknowledgments}

None.

\section{Conflicts of interest}

The authors declare there is no conflict of interests.

\section{Funding}

None.

\section{References}

1. Robert Thong. Root Causes of the Pharmaceutical R\&D Productivity Crisis. Sci Tech Strategy Blogs. 2015.

2. Arrowsmith J. Trial watch:phase III and submission failures:2007-2010. Nat Rev Drug Disco. 2011;10(2):87.

3. Arrowsmith J. Trial watch:phase II failures:2008-2010. Nat Rev Drug Discov. 2011;10(5):328-329. 
4. Munos B. Lessons from 60 years of pharmaceutical innovation. Nat Rev Drug Discov. 2009;8(12):959-968.

5. Cook D, Brown D, Alexander R, et al. Lessons learned from the fate of AstraZeneca's drug pipeline. Nat Rev Drug Discov. 2014;13(6):419431.

6. Milne CP. Academic-Industry Partnerships for Biopharmaceutical Research \& Development:Advancing Medical Science in the US. Tufts CSDD Internal News; 2013:1-26.

7. Janero DR. Positioning for Success in University-Industry DrugDiscovery Collaborations:Initiatives towards Effective TransConstituency Team Science. Int J Drug Dev \& Res. 2015;7(4):60-65.

8. Subrahmanyam VV. Chapter 12:Pathophysiology of endogenous toxins and their relation to in born errors of metabolism and drug mediated toxicities. In: PJ O’Brien, B Bruce, editors. Germany: Wiley-VCH Verlag GmBH \& Co KgaA; 2009:292-316.

9. Subrahmanyam VV, Tonelli AP. Biomarkers, metabonomics and drug development: can inborn errors of metabolism help in understanding drug toxicity? AAPS Journal. 2007;9(3):284-297.

10. Bitensky MW, Kowluru A, Kowluru RA. Non-enzymatic glycation and protein recognition. Prog Clin Biol Res. 1989;304:185-203.

11. Shokrzadeh M, Abdi H, Asadollah PA, et al. Nanoceria Attenuated High Glucose-Induced oxidative damage in HepG2 Cells. Cell J. 2016;18(1):97-102.

12. Scott LJ, Erdos MR, Huyghe JR, et al. The genetic regulatory signature of type 2 diabetes in human skeletal muscle. Nat Commun. 2016;29(7):11764.

13. Hertel JK, Johansson S, Midthjell K, et al. Type 2 diabetes genesPresent status and data from Norwegian studies. Norsk Epidemiologi. 2013;23(1):9-22.

14. Peiró C, Romacho T, Azcutia V, et al. Inflammation, glucose, and vascular cell damage:the role of the pentose phosphate pathway. Cardiovasc Diabetol. 2016;15(1):82.

15. Miller EC, Miller JA. Mechanisms of Chemical Carcinogenesis. Cancer. 1981;47(5):1055-1064.

16. Saga TJ. Overview of Tumor Promotion. Environ Health Perspect. 1983;50:3-14.

17. Petrusevska RT, Fürstenberger G, Marks F, et al. Cytogenetic effects caused by phorbol ester tumor promoters in primary mouse keratinocyte cultures:correlation with the convertogenic activity of TPA in multistage skin carcinogenesis. Carcinogenesis. 1988;9(7):1207-1215.

18. Rushmore TH, Ghazarian DM, Subrahmanyam VV, et al. Probable free radical effects on rat liver nuclei during early hepatocarcinogenesis with a choline-devoid low methionine diet. Cancer Res. 1987;47(24):67316740 .

19. Subrahmanyam VV, Smith MT. Free radical mediated hematopoietic toxicity by drugs, environmental pollutants, and ionizing radiation. In: Wallace KB, editor. Washington DC, USA: CRC Press: A Taylor \& Francis Group; 1997:249-278.
20. Kolachana P, Subrahmanyam VV, Meyer KB, et al. Benzene and its phenolic metabolites produce oxidative DNA damage in HL60 cells in vitro and in the bone marrow in vivo. Cancer Res. 53(5):1023-1026.

21. Vogelstein B, Papadopoulos N, Velculescu VE, et al. Cancer Genome Landscapes. Science. 2013;339(6127):1546-1558.

22. Conrad DF, Keebler JEM, DePristo MA, et al. Variation in genomewide mutation rates within and between human families. Nat Genet. 2011;43(7):712-714.

23. Sérgia V, Cátia M, Luís C, et al. BRAF, KRAS and PIK3CA mutations in colorectal serrated polyps and cancer:Primary or secondary genetic events in colorectal carcinogenesis? BMC Cancer. 2008;8:255.

24. Kirchhoff T, Satagopan JM, Kauff ND, et al. Frequency of BRCA1 and BRCA2 mutations in unselected Ashkenazi Jewish patients with colorectal cancer. J Natl Cancer Inst. 2004;96(1):68-70.

25. Gruber SB, Ellis NA, Scott KK, et al. BLM heterozygosity and the risk of colorectal cancer. Science. 2002;297:5589.

26. Guillem JG, Rapaport BS, Kirchhoff T, et al. A636P is associated with early-onset colon cancer in Ashkenazi Jews. J Am Coll Surg. 2003;196(2):222-225.

27. Peterlongo P, Nafa K, Lerman GS, et al. MSH6 germline mutations are rare in colorectal cancer families. Int J Cancer. 2003;107(4):571-579.

28. Bruce WR, Lee O, Liu Z, et al. Biomarkers of exposure to endogenous oxidative and aldehyde stress. Biomarkers. 2011;16(5):453-456.

29. Kong SY, Takeuchi M, Bruce WR, et al. The Association between Glyceraldehyde-Derived Advanced Glycation End-Products and Colorectal Cancer Risk. Cancer Epidemiol Biomarkers Prev. 2015;24(12):1855-1863.

30. Nicholas W. In the Genome Race, the Sequel Is Personal. The new York times. 2007.

31. We are all mutants: First direct whole-genome measure of human mutation predicts 60 new mutations in each of us. Science Daily.

32. Levy S, Sutton G, Ng PC, et al. The diploid genome sequence of an individual human. PLOs Biology. 2007;5(10):254.

33. Bruder CE, Piotrowski A, Gijsbers AA, et al. Phenotypically Concordant and Discordant Monozygotic Twins Display Different DNA CopyNumber-Variation Profiles. Am J Hum Genet. 2008;82(3):763-771.

34. Arbitrio M, Di Martino MT, Scionti F, et al. DMETTM (Drug Metabolism Enzymes and Transporters):a Pharmacogenomic platform for precision medicine. Oncotarget. 2016.

35. https://en.wikipedia.org/wiki/\$1,000_genome

36. https://en.wikipedia.org/wiki/Human_Metabolome_Database

37. Subrahmanyam VV, Jakir Pinjari, Prashant Patole, et al. Translational Drug Discovery:Integration of computational modeling, medicinal chemistry, pharmacology, DMPK and Toxicology. USA: John Wiley and Sons: New Jersey; 2012:1-54.

38. Orwant R. Dawn of the Zombies. New Scientist, 2006:40-43. 